\title{
Hierarchically Structured Superoleophobic Surfaces with Ultralow Contact Angle Hysteresis
}

\author{
Arun K. Kota, Yongxin Li, Joseph M. Mabry, and Anish Tuteja*
}

Superoleophobic surfaces can repel essentially any contacting liquid, including various low surface tension liquids. A surface is considered superoleophobic if the apparent contact angle $\theta^{*}$ for the contacting liquid droplet is greater than $150^{\circ}$ and the surface displays low contact angle hysteresis $\Delta \theta^{*}$ (the difference between the advancing and receding contact angles). ${ }^{[1]}$ While there are several natural and engineered superhydrophobic surfaces $\left(\theta^{*}>150^{\circ}\right.$ and $\Delta \theta^{*}<5^{\circ}$ for water $)$, there are no naturally occurring superoleophobic surfaces. This is because low surface tension liquids, such as various oils and alcohols, tend to easily wet and spread on most solid surfaces. Superhydrophobic surfaces have been extensively investigated, both experimentally and theoretically, in the past decade. ${ }^{[2-13]}$ In comparison, there are significantly fewer publications on developing superoleophobic surfaces. In recent work, we $\mathrm{e}^{[1,14-17]}$ and others ${ }^{[18-20]}$ explained how re-entrant surface curvature in conjunction with surface chemistry and roughness can be used to design superoleophobic surfaces. While several surfaces have now been engineered with $\theta^{*}>150^{\circ}$ for various low surface tension liquids, none of them, ${ }^{[19-30]}$ including our previous work, ${ }^{[1,14-17]}$ reported a contact angle hysteresis $\Delta \theta^{*}<5^{\circ}$ with liquids possessing a surface tension lower than that of $n$-hexadecane $\left(\gamma_{l v}=\right.$ $\left.27.5 \mathrm{mN} \mathrm{m}^{-1}\right)$. Note that $\Delta \theta^{*}<5^{\circ}$ has been reported before on surfaces that show low apparent contact angles $\left(\theta^{*}<120^{\circ}\right){ }^{[31]}$ However, such surfaces require a lubricating liquid film. Developing superoleophobic surfaces with ultralow contact angle hysteresis can extend the applications of superhydrophobic surfaces to low surface tension liquids, as well as, open avenues to new applications. Such surfaces are expected to be critical for a range of applications including stain-free textiles and spillresistant protective wear, ${ }^{[24]}$ enhanced solvent-resistance, ${ }^{[29]}$ self-cleaning, ${ }^{[9]}$ reduction of biofouling, ${ }^{[32]}$ finger-print resistant surfaces for flat-panel displays, cellphones and sunglasses, ${ }^{[33]}$ drag reduction, ${ }^{[34]}$ developing truly "nonstick" coatings and icephobicity. ${ }^{[35]}$

Contact angle hysteresis is a strong function of the contact area between the solid surface and the liquid droplet. In

Dr. A. K. Kota, Y. Li, Prof. A. Tuteja

Department of Materials Science and Engineering

University of Michigan

Ann Arbor, MI 48109, USA

E-mail: atuteja@umich.edu

Dr. J. M. Mabry

Rocket Propulsion Division

Air Force Research Laboratory

Edwards Air Force Base, CA 93524, USA

DOI: 10.1002/adma.201202554 order to design a surface with low contact angle hysteresis, it is important to lower the solid-liquid contact area. Hierarchically structured surfaces, i.e., surfaces with more than one scale of texture (typically a finer length scale texture on an underlying coarser length scale texture), that can support the so called Cassie-Baxter state, ${ }^{[36]}$ display low contact angle hysteresis with contacting liquids because of the reduced solid-liquid contact area. ${ }^{[10]}$ In this work, we utilize and expand upon the previous advances in understanding superhydrophobic ${ }^{[2-13]}$ and superoleophobic ${ }^{[1,14-20]}$ surfaces to systematically design hierarchically structured superoleophobic surfaces that exhibit ultralow contact angle hysteresis with essentially any contacting liquid. Our surfaces allow, for the first time, even extremely low surface tension liquids, such as $n$-heptane $\left(\gamma_{l v}=20.1 \mathrm{mN} \mathrm{m}^{-1}\right)$, to bounce and roll-off at angles $\omega \leq 2^{\circ}$. Further, we have also studied, for the first time, the influence of systematically varying the interfeature spacing for both the coarser length scale and the finer length scale re-entrant features of the hierarchical surface on the measured contact angle hysteresis.

When a liquid comes in contact with a textured surface, it leads to either the fully wetted Wenzel ${ }^{[37]}$ state or the CassieBaxter $^{[36]}$ state, which supports a composite solid-liquid-air interface. The formation of the Cassie-Baxter state reduces the solid-liquid interfacial area, thereby promoting high apparent contact angles $\theta^{*}$ and low contact angle hysteresis $\Delta \theta^{*} .{ }^{[4,38-40]}$ Development of superoleophobic surfaces requires the design of substrates that promote the formation of the Cassie-Baxter state with essentially any contacting liquid. In our previous work, we $\mathrm{e}^{[1,14-17,41]}$ discussed two dimensionless design parameters, the spacing ratio $D^{*}$ and the robustness factor $A^{*}$, for the systematic design of superoleophobic surfaces.

The spacing ratio $D^{*}$ is a measure of the air trapped underneath a liquid droplet when it forms a composite interface with a textured surface. For textured surfaces composed of discrete spherical particles (such as the electrospun microbeads considered here), $D_{\text {particle }}^{*}=\left[\left(R_{\text {particle }}+D_{\text {particle }}\right) / R_{\text {particle }}\right]^{2}$, where $R_{\text {particle }}$ is the radius of the spherical particle and $D_{\text {particle }}$ is half the interparticle spacing. The Cassie-Baxter relationship can be rewritten in terms of $D_{\text {particle }}^{*}$ as: ${ }^{[16]}$

$\cos \theta_{\text {particle }}^{*}=-1+\frac{1}{D_{\text {particle }}^{*}}\left[\frac{\pi}{2 \sqrt{3}}(1+\cos \theta)\right]^{2}$

Here $\theta$ is the Young's contact angle. ${ }^{[42]}$ Similarly, for textured surfaces composed of cylindrical fibers (such as the stainless steel meshes considered here), $D_{\text {fiber }}^{*}=\left(R_{f i b e r}+D_{f i b e r}\right) / R_{f i b e r}$, where $R_{\text {fiber }}$ is the radius of the cylindrical fiber and $D_{\text {fiber }}$ is half the inter-fiber spacing. The Cassie-Baxter relationship can be rewritten in terms of $D^{*}$ fiber as: ${ }^{[1,14,16]}$ 
$\cos \theta_{\text {fiber }}^{*}=-1+\frac{1}{D_{\text {fiber }}^{*}}[\sin \theta+(\pi-\theta) \cos \theta]$

Now, consider a hierarchically structured surface composed of discrete spherical particles on top of the underlying cylindrical fiber texture. The Cassie-Baxter relationship for such a surface can be rewritten recursively ${ }^{[11,14]}$ as:

$$
\begin{aligned}
\cos \theta_{\text {hierarchical }}^{*}= & -1+\frac{1}{D_{\text {fiber }}^{*}} \\
& \times\left[\sin \theta_{\text {particle }}^{*}+\left(\pi-\theta_{\text {particle }}^{*}\right) \cos \theta_{\text {particle }}^{*}\right]
\end{aligned}
$$

Higher values of $D^{*}$ particle and $D^{*}$ fiber correspond to a higher fraction of air within the composite interface, thereby resulting in a higher $\theta^{*}$ hierarchical (see Equations (1) and (3)).

The robustness factor $A^{*}$ represents the ratio between the breakthrough pressure $P_{\text {breakthrough }}$ required to force the transition from the nonwetting Cassie-Baxter state to the fully wetted Wenzel state and a characteristic reference pressure $P_{\text {ref }}$, given as $P_{r e f}=2 \gamma_{l v} / l_{c a p}$, where $l_{c a p}=\sqrt{\gamma_{v v} / \rho g}$ is the capillary length (here $\gamma_{v}$ is the liquid surface tension, $\rho$ is the liquid density and $g$ is the acceleration due to gravity). This reference pressure $P_{\text {ref }}$ is approximately the minimum possible pressure difference across the composite interface for millimetric or larger liquid droplets or puddles on extremely nonwetting textured surfaces..$^{[1]}$ For textured substrates composed of discrete spherical particles, the robustness factor $A^{*}$ particle is given as: ${ }^{[16]}$

$$
\begin{aligned}
A_{\text {particle }}^{*} & =\frac{P_{\text {breakthrough }}}{P_{\text {ref }}} \\
& =\frac{2 \pi l_{\text {cap }}}{R_{\text {particle }}\left(2 \sqrt{3} D_{\text {particle }}^{*}-\pi\right)} \frac{(1-\cos \theta)}{\left(\sqrt{D_{\text {particle }}^{*}}-1+2 \sin \theta\right)}
\end{aligned}
$$

For textured surfaces composed of cylindrical fibers, the robustness factor $A_{\text {fiber }}^{*}$ is given as: $:^{[1,14,16]}$

$$
\begin{aligned}
A_{\text {fiber }}^{*} & =\frac{P_{\text {breakthrough }}}{P_{\text {ref }}} \\
& =\frac{l_{\text {cap }}}{R_{\text {fiber }}\left(D_{\text {fiber }}^{*}-1\right)} \frac{(1-\cos \theta)}{\left(D_{\text {fiber }}^{*}-1+2 \sin \theta\right)}
\end{aligned}
$$

For a hierarchically structured surface composed of the discrete spherical particles on top of the underlying cylindrical fiber texture, the composite interface is the least stable on the texture with the largest interfeature spacing, ${ }^{[14]}$ i.e., the cylindrical fiber texture (assuming $R_{\text {particle }}<<D_{\text {fiber }}$ ). Consequently, the robustness factor for the hierarchically structured surface, $A^{*}$ hierarchical $=A_{\text {fiber }}^{*}$. Large values of robustness factor $\left(A^{*}\right.$ hierarchical $\left.\gg 1\right)$ indicate the formation of a robust composite interface with very high breakthrough pressures. On the other hand, as $A^{*}{ }_{\text {hierarchical }}$ approaches unity, $P_{\text {breakthrough }}$ decreases towards $P_{\text {ref. }}$. Thus, a composite interface on any surface with $A^{*}$ hierarchical $<1$ cannot maintain its stability against even small pressure differentials, causing the liquid to penetrate into the textured surface and ultimately leading to the fully wetted Wenzel state.
In this work, we employed a single-step technique, based on electrospinning microbeads (finer length scale texture) of $50 \mathrm{wt} \%$ fluorodecyl POSS ${ }^{[1,15]}+$ PMMA (poly(methylmethacrylate)) blend $\left(\gamma_{s v}=10.3 \mathrm{mN} \mathrm{m}^{-1}\right)$ onto textured substrates (stainless steel wire meshes) possessing re-entrant curvature on the coarser length scale (see the Experimental Section). Note that the microbeads completely cover the wire mesh, i.e., they are coated all around each wire. This allows us to fabricate novel hierarchically structured surfaces that possess re-entrant curvature on both the coarser and the finer length scales.

In order to systematically investigate the influence of the finer length scale texture on the apparent contact angle $\theta^{*}$ and the contact angle hysteresis $\Delta \theta^{*}$ for various low surface tension liquids, we electrospun microbeads of $50 \mathrm{wt} \%$ fluorodecyl POSS + PMMA blend onto a nontextured substrate (silicon wafer). We used four different solvents for the electrospinning process-Asahiklin 225 (AK225) only, as well as, mixtures of AK225 with 5, 10, and 15 vol\% dimethyl formamide (DMF). The highly porous structure obtained using AK225 only (Figure $1 \mathrm{a}, R_{\text {particle }} \approx 10 \mu \mathrm{m}, D_{\text {particle }}^{*}=30.3$ ) results in a high apparent contact angle $\theta_{\text {oil, adv }}^{*}=163^{\circ}$ for $n$-hexadecane. However, the same structure is completely wet by $n$-heptane $\left(\theta^{*}{ }_{\text {oil ad }} \approx 0^{\circ}\right.$, see Figure 1e). This is because, even though the fabricated texture can support $n$-hexadecane $\left(\theta_{\text {oil,adv }}=79^{\circ}\right.$, $\left.A_{\text {particle }}^{*}=1.5\right)$ in the Cassie-Baxter state, it allows $n$-heptane $\left(\theta_{\text {oil,adv }}=61^{\circ}, A_{\text {particle }}^{*}=0.9\right)$ to transition to the Wenzel state. As the vol\% of DMF in the solution increases, the spacing between the microbeads decreases (see Figure 1b-d), which in turn leads to an increase in the value of robustness factor $A^{*}$ particle. Consequently, the microbead structure obtained using a mixture of AK225 with 5 vol\% DMF (Figure $1 \mathrm{~b}, R_{\text {particle }} \approx 6.7 \mu \mathrm{m}, D_{\text {particle }}^{*}=$ $15.9)$ can support both $n$-hexadecane $\left(A_{\text {particle }}^{*}=5.6\right)$ and $n$-heptane $\left(A_{\text {particle }}^{*}=3.4\right)$ in the Cassie-Baxter state with a reasonably low contact angle hysteresis $\left(\Delta \theta^{*}{ }_{\text {oil }}=6^{\circ}\right.$ for $n$-hexadecane and $\Delta \theta^{*}{ }_{\text {oil }}=10^{\circ}$ for $n$-heptane). While morphologies obtained using higher DMF content can also support both $n$-hexadecane and $n$-heptane in the Cassie-Baxter state, the lower porosity (i.e., higher solid-liquid contact area) leads to a significantly higher contact angle hysteresis $\Delta \theta^{*}$ oil . Thus, we utilized the structure obtained using a mixture of AK225 with 5 vol\% DMF as the finer length scale texture in all the hierarchically structured surfaces discussed below.

Figure 2a shows a stainless steel mesh $70\left(D^{*}\right.$ fiber $\left.=1.4\right)$ uniformly coated with electrospun microbeads of $50 \mathrm{wt} \%$ fluorodecyl POSS + PMMA blend. This hierarchically structured surface possesses re-entrant texture on both the coarser and finer length scales. The hierarchical texture, along with the low solid surface energy, renders our surfaces superoleophobic with high apparent contact angles (see Figure $2 \mathrm{~b}$ and section 1 in the supporting information) and ultralow contact angle hysteresis even with extremely low surface tension liquids such as $n$-heptane $\left(\theta^{*}{ }_{\text {oil, adv }}=155^{\circ}, \Delta \theta^{*}{ }_{\text {oil }}=4^{\circ}\right)$. To our knowledge, this is the first-ever report of a superoleophobic surface that demonstrates a contact angle hysteresis $<10^{\circ}$ with an extremely low surface tension liquid such as $n$-heptane. Note that the contact angle hysteresis for $n$-heptane on a surface with the coarser length scale texture only (stainless steel mesh 70 dipcoated with $50 \mathrm{wt} \%$ fluorodecyl POSS + PMMA blend) is $\Delta \theta^{*}$ oil $=105^{\circ}$ and on a surface with the finer length scale texture only 

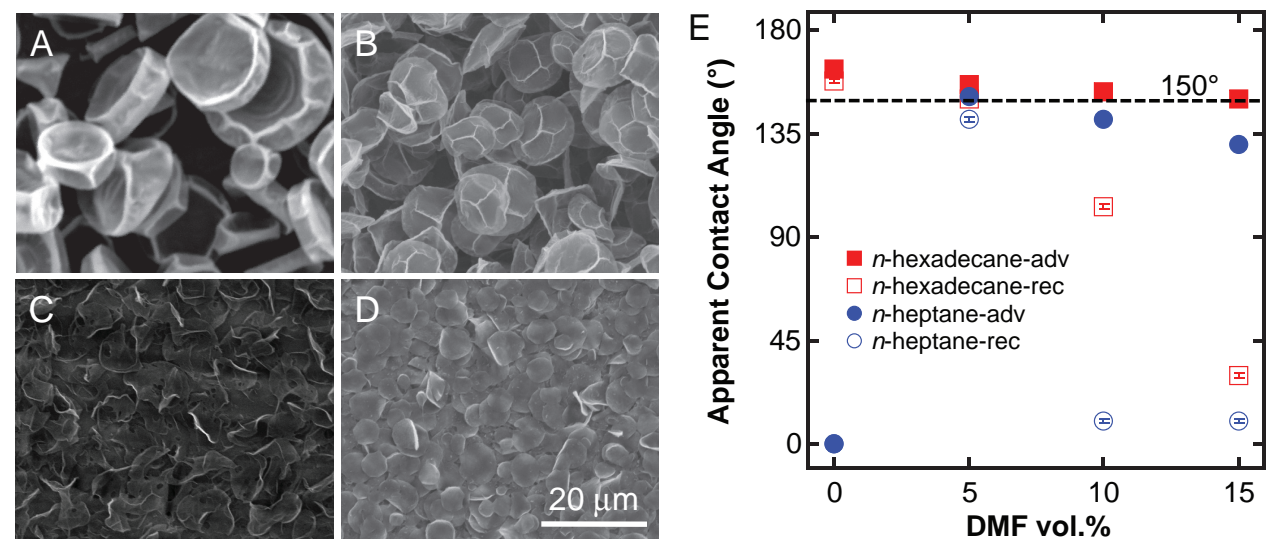

Figure 1. a), b), c), and d) SEM images of electrospun microbeads of 50 wt\% fluorodecyl POSS + PMMA blend obtained using AK225 only, AK225 with 5 vol\% DMF, AK225 with 10 vol\% DMF, and AK225 with 15 vol\% DMF, respectively. The substrates for electrospinning are (nontextured) silicon wafers. e) The advancing and receding apparent contact angles for $n$-hexadecane and $n$-heptane on the surfaces shown in a)-d).

(electrospun microbeads of $50 \mathrm{wt} \%$ fluorodecyl POSS + PMMA blend on a silicon wafer) is $\Delta \theta^{*}{ }_{\text {oil }}=10^{\circ}$ (Figure 2c). The ultralow contact angle hysteresis on the hierarchically structured sur- face is a direct consequence of the reduced solid-liquid contact area. Indeed, an inspection of the vicinity of the triple phase contact line (see the Experimental Section) shows a reduction

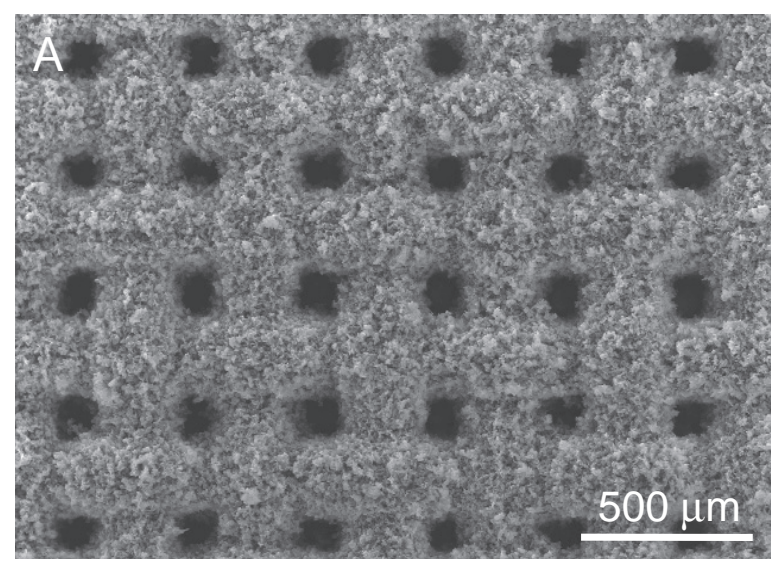

○

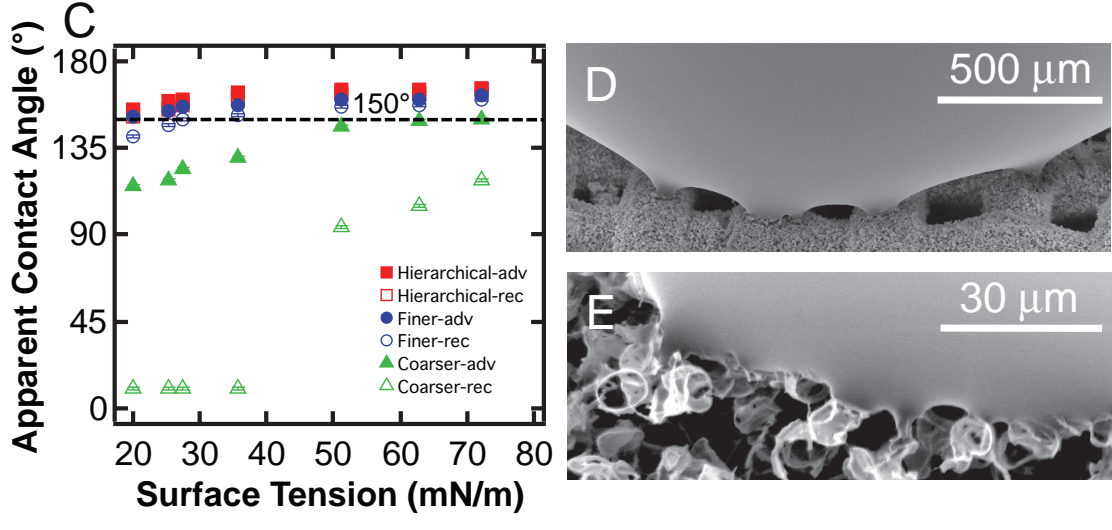

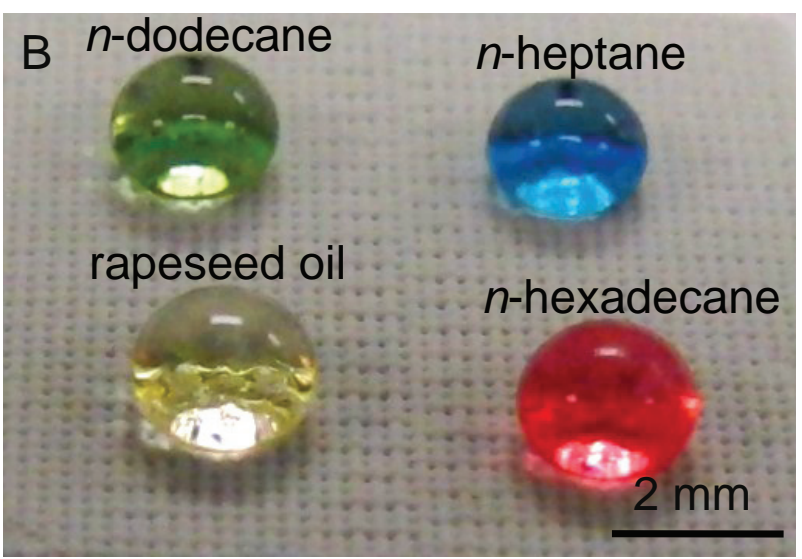

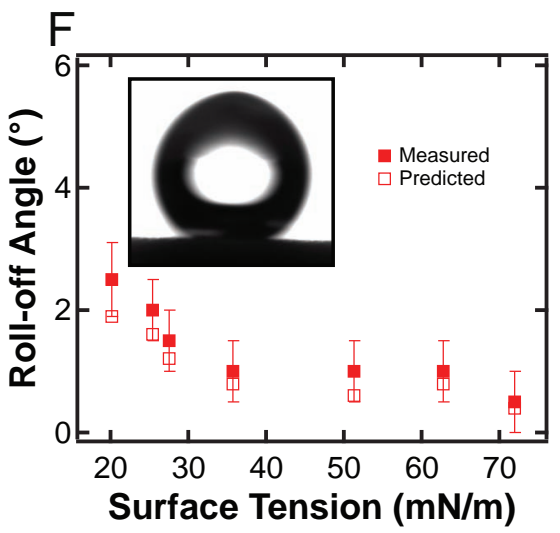

Figure 2. a) SEM image of a stainless steel mesh 70 uniformly coated with electrospun microbeads of 50 wt $\%$ fluorodecyl POSS + PMMA blend. b) Droplets of rapeseed oil $\left(\gamma_{\nu}=35.7 \mathrm{mN} \mathrm{m}^{-1}\right), n$-hexadecane $\left(\gamma_{\nu}=27.5 \mathrm{mN} \mathrm{m}^{-1}\right), n$-dodecane $\left(\gamma_{\nu}=25.3 \mathrm{mN} \mathrm{m}^{-1}\right)$ and $n$-heptane $\left(\gamma_{\nu}=20.1 \mathrm{mN} \mathrm{m} \mathrm{m}^{-1}\right)$ showing very high apparent contact angles on the surface shown in a). c) Advancing and receding apparent contact angles for various liquids on a hierarchically structured surface, as well as, on surfaces with the coarser length scale texture only and on surfaces with the finer length scale texture only. The receding contact angle legends are not visible for the hierarchically structured surfaces because of the ultralow contact angle hysteresis. d) and e) SEM images showing the vicinity of the contact line along the coarser length scale texture and the finer length scale texture, respectively, of the hierarchically structured surface. The distortions in the contact line are evidence of air trapped at both the length scales. $\mathrm{f}$ ) Roll-off angles of various liquids on the hierarchically structured surface. The inset shows a $n$-heptane droplet rolling on a hierarchical surface at a roll-off angle $\omega=3^{\circ}$. 


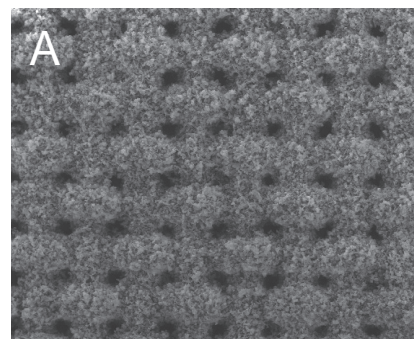

$\mathrm{E}$

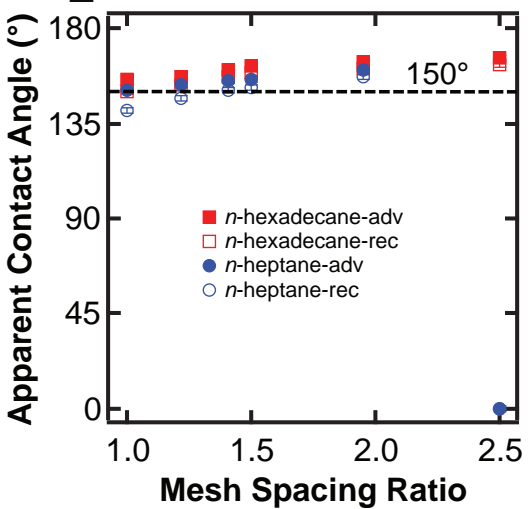

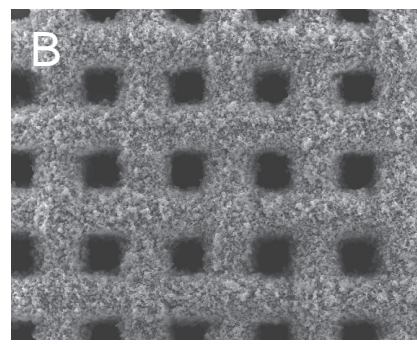

$\mathrm{F}$

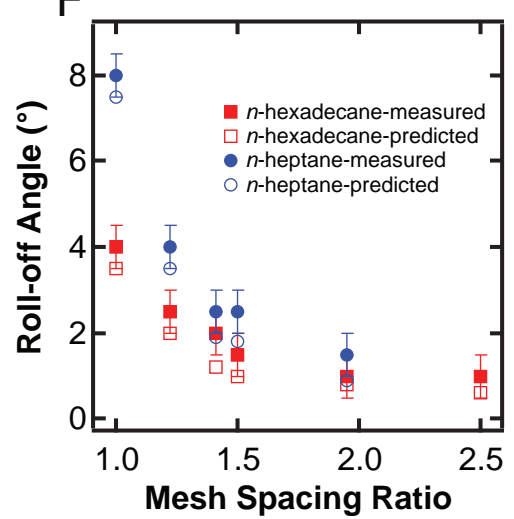

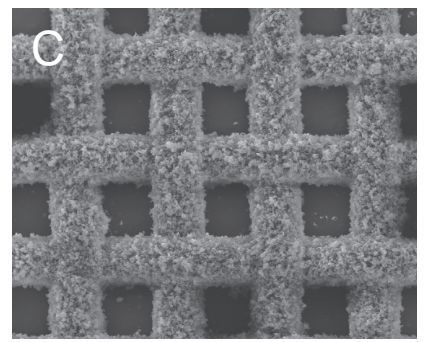

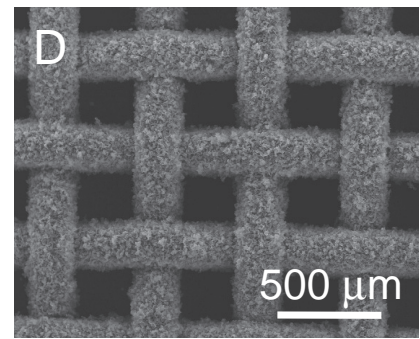

$\mathrm{G}$

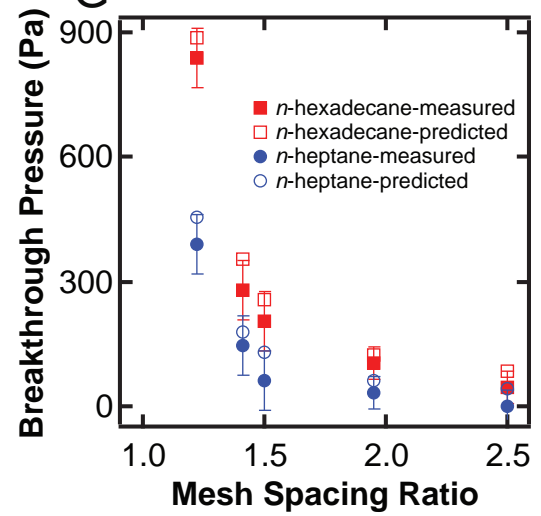

Figure 3. a), b), c) and d) SEM images of the hierarchically structured surfaces with a mesh spacing ratio $D_{\text {fiber }}^{*}=1.2, D_{\text {fiber }}^{*}=1.5, D_{\text {fiber }}^{*}=1.9$, and $D^{*}{ }_{\text {fiber }}=2.5$, respectively. e), $\mathrm{f}$ ) and g) The apparent contact angles, the roll-off angles, and the breakthrough pressures, respectively, as a function of the mesh spacing ratio for $n$-hexadecane and $n$-heptane.

of the solid-liquid contact area due to the air trapped along both the coarser length scale texture (Figure 2d), as well as the finer length scale texture (Figure 2e). This ultralow contact angle hysteresis results in roll-off angles $\omega \leq 3^{\circ}$ for essentially any contacting liquid with surface tension $\gamma_{v} \geq 20.1 \mathrm{mN} \mathrm{m}^{-1}$ (Figure 2f). Note that all our roll-off angle measurements utilized very small $(\approx 2 \mu \mathrm{L})$ liquid droplets. The experimentally measured roll-off angles match reasonably well with our predictions (see Supporting Information, Section 2). Videos illustrating the roll-off of $n$-hexadecane (Movie S1) and $n$-heptane (Movie S2) droplets on a stainless steel mesh 70 coated with electrospun microbeads of $50 \mathrm{wt} \%$ fluorodecyl POSS + PMMA blend are included in the Supporting Information. To our knowledge, this is the first-ever report of $n$-heptane droplets rolling off from a surface at such low tilt angles.

In order to systematically study the influence of the coarser length scale texture on the contact angle hysteresis, we prepared different hierarchically structured surfaces with increasing mesh spacing ratio $D_{\text {fiber }}^{*}$ (Figures $3 \mathrm{a}-\mathrm{d}$ ). As $D_{\text {fiber }}^{*}$ increases, the solid-liquid contact area decreases (or the fraction of air trapped underneath the liquid droplet increases). Consequently, the apparent contact angles increase, the contact angle hysteresis decreases (Figure 3e), and the roll-off angles decrease (Figure 3f) with increasing $D^{*}$ fiber Indeed, for $D^{*}$ fiber $=1.9$, we obtain $\theta^{*}{ }_{\text {oil }, a d v}=160^{\circ}, \Delta \theta^{*}{ }_{\text {oil }}=3^{\circ}$, and $\omega \leq 2^{\circ}$ for $n$-heptane. The exceptionally low values of contact angle hysteresis and roll-off angles for liquids, such as $n$-heptane, illustrate the uniqueness of our hierarchically structured surfaces. These results are particularly interesting given that the $50 \mathrm{wt} \%$ fluorodecyl POSS + PMMA blend used to fabricate our superoleophobic surfaces is inherently oleophilic, i.e., it possesses a Young's contact angle significantly less than $90^{\circ}$ for $n$-heptane $\left(\theta_{a d v}=61^{\circ}\right.$ and $\theta_{\text {rec }}$ $=38^{\circ}$; see Supporting Information, Section 1 ). At very high values of $D^{*}{ }_{\text {fiber }}$ however, the surface can no longer support the contacting liquid in the Cassie-Baxter state because the value of the robustness factor $A^{*}$ hierarchical decreases rapidly. For example, when $D^{*}$ fiber $=2.5, n$-heptane $\left(A^{*}\right.$ hierarchical $\left.=1.8\right)$ breaks through and shows a very low apparent contact angle. Figure $3 \mathrm{~g}$ shows the breakthrough pressure, $P_{\text {breakthrough }}$, as a function $D_{\text {fiber }}^{*}$ for both $n$-heptane and $n$-hexadecane. It is evident that the experimentally measured values match reasonably well with those predicted using Equation (5).

When a liquid droplet of density $\rho$ is dropped under gravity from a height $h$, it impacts the solid surface with a hydrostatic pressure $P_{\text {applied }}=\rho g h$. We observed that essentially all liquid droplets with surface tension $\gamma_{l v} \geq 20.1 \mathrm{mN} \mathrm{m}^{-1}$ impacting our hierarchical surface bounced back, as long as $P_{\text {applied }}<P_{\text {breakthrough }}$. When $2 \mu \mathrm{L} n$-heptane droplets (radius $\approx 0.8 \mathrm{~mm}$ ) were dropped on the hierarchically structured surface (with $P_{\text {breakthrough }}=34 \mathrm{~Pa}$ ) from a height $h=3 \mathrm{~mm}\left(P_{\text {applied }}=20.1 \mathrm{~Pa}\right)$ above the surface, they bounced at least four times before rolling off from the surface (Figure 4). This indicates that liquids, such as $n$-heptane (with $\theta_{o i l}<<90^{\circ}$ ), form a robust metastable Cassie-Baxter state on our hierarchically structured surfaces, as parameterized by the high values of $P_{\text {breakthrough }}$. The coefficient of restitution for the bounce was as high as 0.86 and 0.92 for $n$-hexadecane and $n$-heptane, respectively. This further affirms the ultralow contact angle hysteresis of our surfaces. To our knowledge, this is the first-ever report of a superoleophobic surface that allows even extremely low surface tension liquids, such as $n$-heptane, 


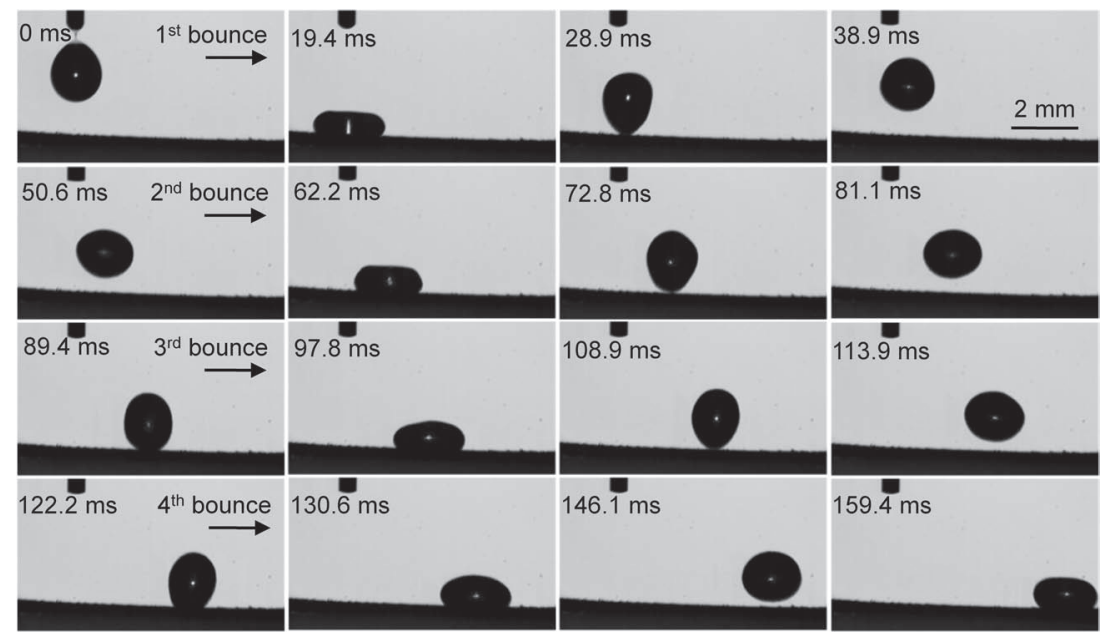

Figure 4. A series of snapshots showing a $2 \mu \mathrm{L} n$-heptane droplet bouncing on a hierarchically structured surface (stainless steel mesh 70 uniformly coated with electrospun microbeads of $50 \mathrm{wt} \%$ fluorodecyl POSS + PMMA blend) that is tilted $2^{\circ}$ relative to the horizontal plane. The droplet was dropped from a height $h=3 \mathrm{~mm}$ above the surface, i.e., the impact velocity is $0.24 \mathrm{~m} \mathrm{~s}^{-1}$. The droplet bounces four times before rolling off from the surface (also see Movie S4).

to bounce back. Videos illustrating the bouncing of two low surface tension liquids, $n$-hexadecane (Movie S3) and n-heptane (Movie S4), are included in the Supporting Information. It is evident from the movies that the deformation in the shape of the $n$-heptane droplet during bouncing is significantly greater than that for the $n$-hexadecane droplet. This is because of the lower surface tension and lower viscosity of $n$-heptane when compared with $n$-hexadecane. ${ }^{[43]}$

The mechanical durability of different superoleophobic surfaces is critical for their commercial application. Developing durable superoleophobic surfaces has thus far been a significant challenge. While our hierarchical superoleophobic surfaces retain their superoleophobicity when exposed to harsh environments such as ozone or $254 \mathrm{~nm}$ UV light, their mechanical durability still needs to be improved. One way of potentially improving the mechanical durability of our hierarchical structures is to anneal the surfaces above the glass transition temperature of PMMA. Our experiments show that such thermal annealing improves the adhesion of the electrospun coatings to the underlying substrate and also creates a robust connected network. However, such an annealing step needs to be optimized so that the robust connected network is not realized at the cost of reducing the porosity of the hierarchical structure, which can significantly reduce the observed apparent contact angles.

In conclusion, we have developed a simple, single-step technique, based on electrospinning microbeads onto textured surfaces, to develop re-entrant, hierarchically structured, superoleophobic surfaces. We demonstrate that we can tune the contact angle hysteresis on these hierarchical surfaces by systematically varying the interfeature spacing for both the coarser length scale and the finer length scale features. The low surface energy and the significantly reduced solid-liquid contact area allow our hierarchically structured surfaces to exhibit ultralow contact angle hysteresis even for extremely low surface tension liquids such as $n$-heptane (with $\theta_{\text {oil }}<90^{\circ}$ ). This ultralow contact angle hysteresis allows, for the first time, droplets of essentially any contacting liquid, including $n$-heptane, to easily roll-off (roll-off angles $\omega \leq 2^{\circ}$ ) and bounce on our surfaces. This result is particularly interesting given that the materials used to fabricate the superoleophobic surfaces are inherently oleophilic.

\section{Experimental Section}

Electrospinning, Dip-coating and Spin-coating: $5 \mathrm{mg} \mathrm{mL} \mathrm{m}^{-1}$ solutions of $50 \mathrm{wt} \% 1 \mathrm{H}, 1 \mathrm{H}, 2 \mathrm{H}, 2 \mathrm{H}$ heptadecafluorodecyl polyhedral oligomeric silsequioxane (fluorodecyl POSS) [15] + poly(methylmethacrylate) (PMMA, $M_{w} \approx 35000 \mathrm{~g} \mathrm{~mol}^{1}$ ) were prepared in Asahiklin 225 (AK225, Asahi Glass Co.) only, as well as, in mixtures of AK225 with 5, 10 , and 15 vol\% dimethyl formamide (DMF). The solutions were electrospun using a custom-built apparatus, at a flow rate, voltage and plate-toplate distance of $30 \mathrm{~mL} \mathrm{~min}{ }^{-1}, 15 \mathrm{kV}$ and $25 \mathrm{~cm}$, respectively. Hierarchically structured surfaces were prepared by electrospinning the $50 \mathrm{wt} \%$ fluorodecyl POSS + PMMA solutions in AK225 with 5 vol\% DMF onto stainless steel wire meshes (McMaster Carr) of mesh sizes 300,100,70,60, and 50. Surfaces with texture on the finer length scale only were prepared by electrospinning each of the $50 \mathrm{wt} \%$ fluorodecyl POSS + PMMA solutions onto silicon wafers. Surfaces with the coarser length scale texture only were prepared by dip-coating the stainless steel wire meshes with the $50 \mathrm{wt} \%$ fluorodecyl POSS + PMMA solutions in AK225 for 10 min and subsequently drying in air. Nontextured surfaces were prepared by spin-coating silicon wafers with $50 \mathrm{wt} \%$ fluorodecyl POSS + PMMA solutions of AK-225 at 1500 RPM for $30 \mathrm{~s}$. We used the Owens-Wendt approach ${ }^{[4]}$ to estimate the solid surface energy.

Contact Angle and Roll-off Angle Measurements: The contact angle and roll-off angle measurements were conducted using a Ramé-Hart 200-F1 goniometer. The contact angles were measured by advancing or receding a small volume of liquid $(\approx 2 \mu \mathrm{L})$ onto the surface using a 2 $\mathrm{mL}$ micrometer syringe (Gilmont). The roll-off angles were measured by tilting the stage until the $(\approx 2 \mu \mathrm{L})$ droplet rolled off from the surface. At least six measurements were performed on each surface. The errors in contact angle and roll-off angle measurements were $\pm 1^{\circ}$ and $\pm 0.5^{\circ}$, respectively. We used Equation (1), (2), (4), and (5) in conjunction with the contact angle measurements on nontextured substrates (see Supporting Information, Section 1) to estimate $D_{\text {fiben }}^{*} D_{\text {particle, }}^{*} A_{\text {fiben }}^{*}$ and $A_{\text {particle, respectively. }}^{*}$

Microscopy: The surfaces were imaged using a Philips XL30 scanning electron microscope (SEM) at $5 \mathrm{kV}$. We obtained the average size of the electrospun microbeads $\left(R_{\text {particle }} \approx 10 \mu \mathrm{m}\right.$ in Figure $1 \mathrm{a}$ and $R_{\text {particle }}$ $\approx 6.7 \mu \mathrm{m}$ in Figure $1 \mathrm{~b}$ ) using image analysis with Image). Vicinity of the contact line was imaged using a PDMS (resin:hardener ratio was 10:1) droplet that was cross-linked at $70^{\circ} \mathrm{C}$ for $2 \mathrm{~h}$ on top of the hierarchically textured superoleophobic surface. ${ }^{[45]}$

Breakthrough Pressure: Breakthrough pressure was measured using a custom-built apparatus with the hierarchical surface sandwiched between two glass tubes. The upper tube was filled with a liquid column until the liquid breaksthrough the surface and permeates into the lower tube. The hydrostatic pressure corresponding to the maximum liquid column height that the surface withstands is reported as the breakthrough pressure.

Bouncing Droplet Imaging: High-speed movies were obtained using a Fastec Hispecl camera at 2000 frames per second.

\section{Supporting Information}

Supporting Information is available from the Wiley Online Library or from the author. 


\section{Acknowledgements}

A.K.K. and Y.L contributed equally to this work. We thank Dr. Charles Y-C. Lee and the Air Force Office of Scientific Research (AFOSR) for financial support under grants FA9550-11-1-0017 and LRIR-12RZ03COR. We also thank Dr. Sean Ramirez for providing samples of fluorodecyl POSS. Y.L. would like to thank the China Scholarship Council for financial support.

Received: June 23, 2012

Revised: July 29, 2012

Published online: August 29, 2012

[1] A. Tuteja, W. Choi, J. M. Mabry, G. H. McKinley, R. E. Cohen, Proc. Natl. Acad. Sci. USA 2008, 105, 18200.

[2] L. C. Gao, T. J. McCarthy, Langmuir 2006, 22, 2966.

[3] J. Genzer, K. Efimenko, Science 2000, 290, 2130.

[4] A. Lafuma, D. Quere, Nat. Mater. 2003, 2, 457.

[5] A. Marmur, Langmuir 2004, 20, 3517.

[6] W. Ming, D. Wu, R. van Benthem, G. de With, Nano Lett. 2005, 5, 2298.

[7] N. A. Patankar, Langmuir 2003, 19, 1249.

[8] D. Quere, Rep. Prog. Phys. 2005, 68, 2495.

[9] T. L. Sun, L. Feng, X. F. Gao, L. Jiang, Acc. Chem. Res. 2005, 38, 644.

[10] B. Bhushan, Y. C. Jung, Prog. Mater. Sci. 2011, 56, 1.

[11] S. Herminghaus, Europhys. Lett. 2007, 79.

[12] W. Li, A. Amirfazli, J. Colloid Interface Sci. 2005, 292, 195.

[13] W. Li, A. Amirfazli, Adv. Colloid Interface Sci. 2007, 132, 51.

[14] W. Choi, A. Tuteja, S. Chhatre, J. M. Mabry, R. E. Cohen, G. H. McKinley, Adv. Mater. 2009, 21, 2190.

[15] A. Tuteja, W. Choi, M. Ma, J. M. Mabry, S. A. Mazzella, G. C. Rutledge, G. H. McKinley, R. E. Cohen, Science 2007, 318, 1618.

[16] S. S. Chhatre, W. Choi, A. Tuteja, K. C. Park, J. M. Mabry, G. H. McKinley, R. E. Cohen, Langmuir 2010, 26, 4027.

[17] A. Tuteja, W. Choi, G. H. McKinley, R. E. Cohen, M. F. Rubner, MRS Bull. 2008, 33, 752 .

[18] A. Marmur, Langmuir 2008, 24, 7573.
[19] A. Ahuja, J. A. Taylor, V. Lifton, A. A. Sidorenko, T. R. Salamon, E. J. Lobaton, P. Kolodner, T. N. Krupenkin, Langmuir 2008, 24, 9.

[20] L. Cao, T. P. Price, M. Weiss, D. Gao, Langmuir 2008, 24, 1640.

[21] K. Tsujii, T. Yamamoto, T. Onda, S. Shibuichi, Angew. Chem. Int. Ed. 1997, 36, 1011

[22] H. J. Li, X. B. Wang, Y. L. Song, Y. Q. Liu, Q. S. Li, L. Jiang, D. B. Zhu, Angew. Chem. Int. Ed. 2001, 40, 1743

[23] C. T. Hsieh, F. L. Wu, W. Y. Chen, J. Phys. Chem. C 2009, 113 13683.

[24] B. X. Leng, Z. Z. Shao, G. de With, W. H. Ming, Langmuir 2009, 25, 2456.

[25] A. Steele, I. Bayer, E. Loth, Nano Lett. 2009, 9, 501.

[26] D. A. Wang, X. L. Wang, X. J. E. Liu, F. Zhou, J. Phys. Chem. C 2010 114, 9938

[27] T. Fujii, Y. Aoki, H. Habazaki, Langmuir 2011, 27, 11752.

[28] T. Darmanin, F. Guittard, S. Amigoni, E. T. de Givenchy, X. Noblin, R. Kofman, F. Celestini, Soft Matter 2011, 7, 1053.

[29] J. P. Zhang, S. Seeger, Angew. Chem. Int. Ed. 2011, 50, 6652.

[30] X. Deng, L. Mammen, H.-J. r. Butt, D. Vollmer, Science 2011.

[31] T.-S. Wong, S. H. Kang, S. K. Y. Tang, E. J. Smythe, B. D. Hatton, A. Grinthal, J. Aizenberg, Nature 2011, 477, 443.

[32] J. Genzer, K. Efimenko, Biofouling 2006, 22, 339.

[33] T. Verho, C. Bower, P. Andrew, S. Franssila, O. Ikkala, R. H. A. Ras, Adv. Mater. 2011, 23, 673.

[34] C. Lee, C.-J. Kim, Phys. Rev. Lett. 2011, 106.

[35] M. Ma, R. M. Hill, Curr. Opin. Colloid Interface Sci. 2006, 11, 193.

[36] A. B. D. Cassie, S. Baxter, Trans. Faraday Soc. 1944, 40, 0546.

[37] R. N. Wenzel, Ind. Eng. Chem. 1936, 28, 988.

[38] C. W. Extrand, Langmuir 2002, 18, 7991

[39] B. He, N. A. Patankar, J. Lee, Langmuir 2003, 19, 4999.

[40] W. Chen, A. Y. Fadeev, M. C. Hsieh, D. Oner, J. Youngblood, T. J. McCarthy, Langmuir 1999, 15, 3395.

[41] G. Kwon, A. K. Kota, Y. Li, A. Sohani, J. M. Mabry, A. Tuteja, Adv. Mater. 2012, 24, 3666.

[42] T. Young, Philos. Trans. R. Soc. London 1805, 95, 65.

[43] C. Clanet, C. Beguin, D. Richard, D. Quere, J. Fluid Mech. 2004, 517, 199.

[44] D. K. Owens, R. C. Wendt, J. Appl. Polym. Sci. 1969, 13, 1741.

[45] W. Choi, A. Tuteja, J. M. Mabry, R. E. Cohen, G. H. McKinley, J. Colloid Interf. Sci. 2009, 339, 208. 